\title{
Funciones ejecutivas y rendimiento académico en estudiantes de bachillerato dependiente de una casa hogar
}

Executive functions and academic achievement in students from a baccalaureate integrated to an orphanage Funções executivas e desempenho acadêmico em estudantes do ensino
médio dependentes de uma casa

Leonardo Manriquez-López

Universidad Autónoma del Estado de Morelos, México

leonardo.manriquezl@uaem.edu.mx https://orcid.org/0000-0002-3453-8648

\section{Resumen}

Aunque se ha investigado la relación entre las funciones ejecutivas (FE) y el rendimiento académico (RA) en diferentes etapas vitales, no hay ninguna sobre dicha relación en el nivel medio superior; lo cual es trascendente por incluir una época vital de transición para el desarrollo neuropsicológico frontal. Esto se indago en el presente trabajo. Para ello se aplicó la Batería Neuropsicológica de Funciones Ejecutivas a 50 estudiantes de un bachillerato dependiente de una casa hogar, que cuenta con población interna y externa. Se realizaron análisis de correlación y comparación intragrupales, de tipo no paramétrico, entre las dimensiones y tareas de funcionamiento ejecutivo, las medidas de rendimiento académico, y la condición de los estudiantes (internos vs externos) y otras variables demográficas. Se encontraron correlaciones distintas entre las FE de Inhibición, Memoria de Trabajo y Flexibilidad Mental, con los tipos de RA (álgebra, expresión oral y escrita, y tronco común) y con las variables demográficas (género, especialidad y condición); sin embargo, los análisis intragrupales por niveles de desempeño (bajo, medio y alto) arrojaron menos relaciones de significancia entre las variables; siendo las diferencias intragrupales en el RA en álgebra el resultado más constante. 
Palabras clave: bachilleres, desarrollo neuropsicológico, funciones ejecutivas, neuroeducación, rendimiento académico.,

\section{Abstract}

Although the relationship between executive functions (EF) and academic achievement (AA) at different stages of life has been investigated, there is no research on this relationship at the upper secondary level. This is important because the transition for the frontal neuropsychological development in this period of life. This was investigated in the present work. For this, the Batería Neuropsicológica de Funciones Ejecutivas was applied to 50 students from a baccalaureate integrated to an orphanage. Non-parametric intra-group correlation and comparative analysis were carried out between the dimensions and the tasks of executive functioning, the measures of academic performance, the condition of the students and other demographic variables. Significant correlations were found between inhibition, Working Memory and Mental Flexibility, and AA categories and demographic variables; however, the intra-group analyzes by performance levels showed less significant relationships between the variables. The correlations and intra-group differences in algebra AA are the most constant result.

Keywords: academic achievement, high school, neuropsychological development, executive functions.

\section{Resumo}

Embora a relação entre funções executivas (EF) e desempenho acadêmico (AR) em diferentes fases da vida tenha sido investigada, não há relação entre essa relação no nível médio superior; que é transcendente porque inclui um período de transição vital para o desenvolvimento neuropsicológico frontal. Isso é investigado no presente trabalho. Para este fim, a bateria neuropsicológica de funções executivas foi aplicada a 50 alunos de um bacharelado dependente de uma casa, que tem uma população interna e externa. Realizou-se correlação intra-grupo e análise de comparação, não paramétrica, entre as dimensões e tarefas do funcionamento executivo, medidas de desempenho acadêmico e a condição de alunos (internos versus externos) e outras variáveis demográficas. Diferentes correlações foram 


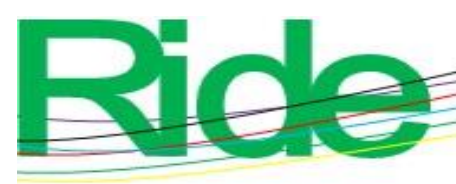

Revista Iberoamericana para la Investigación y el Desarrollo Educativo ISSN 2007 - 7467

encontradas entre a FE de Inibição, Memória de Trabalho e Flexibilidade Mental, com os tipos de AR (álgebra, expressão oral e escrita e tronco comum) e com as variáveis demográficas (gênero, especialidade e condição); entretanto, as análises intragrupo por níveis de desempenho (baixo, médio e alto) mostraram relações menos significativas entre as variáveis; sendo as diferenças intragrupos na AR em álgebra o resultado mais constante.

Palavras-chave: bachilleres, desenvolvimento neuropsicológico, funções executivas, neuroeducação, desempenho acadêmico.

Fecha Recepción: Junio 2018

Fecha Aceptación: Noviembre 2018

\section{Introducción}

En México, y en otras partes del mundo, existe una clara problemática, de gran impacto social, con relación al rendimiento académico (RA) y los niveles de deserción escolar de los estudiantes de nivel bachillerato (Secretaría de Educación Pública [SEP], 2012) y, aunque se ha investigado el rendimiento de los bachilleres con relación a variables, sociales, institucionales y personales (Caso-Niebla y Hernández-Guzmán, 2010), es sorprendente lo poco que se ha indagado en cuanto al aspecto neuropsicológico (Anderson, Anderson, Northam, Jacobs y Catroppa, 2001), sobre todo si asumimos que, en esta etapa se ve acelerada las áreas frontales -consideradas las responsables de la planeación, regulación y verificación de la actividad (Luria 1979/2000) — asociadas al funcionamiento ejecutivo (Casey, Jones y Somerville, 2011) que, teóricamente juega un papel crucial en el aprendizaje y desempeño escolar (Welsh, Peterson y Jameson, 2017).

Se considera que las funciones ejecutivas (FE) están implicadas con "la capacidad de ejecutar un comportamiento no estereotipado en respuesta a demandas específicas del ambiente (Garon, Bryson y Smith, 2008; como se citó en Stelzer y Cervigni, 2011); debido a ello permiten la regulación de la conducta inhibiendo las respuestas automáticas, regulando el desempeño cognitivo, afectivo y conductual y llevando a cabo acciones planificadas para la solución de las problemáticas vitales en turno (Luria, 1979/2000; Stelzer y Cervigni, 2011). 


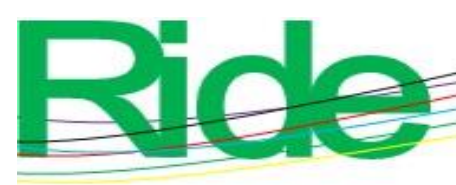

Revista Iberoamericana para la

Investigación y el Desarrollo Educativo

ISSN 2007 - 7467

Los procesos cognitivos que forman parte de las FE y la manera en que interactúan ParaStelzer y Cervigni (2011), existen modelos en los que hay un proceso de control central que modula otros suprocesos; modelos en los que las FE agrupan procesos cognitivos independientes; y modelos mixtos con un componente central y subcomponentes parcialmente independientes. Esto bien podría explicar por qué, aunque parece haber consenso sobre la presencia de una relación entre tales áreas de desempeño, existen grandes diferencias con relación a las relaciones específicas entre los componentes de las FE y el rendimiento en diferentes dominios académicos (Stelzer y Cervigni, 2011).

Las investigaciones de Bryce, Whitebread y Szücs (2015) señalan, que el control inhibitorio y la memoria de trabajo contribuyen de manera importante a dicho rendimiento en niños y adolescentes iniciales, además se han encontrado relaciones entre el cambio atencional, la comprensión de falsas creencias, la flexibilidad cognitiva, la planificación y, la monitorización, con dominios académicos como la lectura, la solución de problemas, las ciencias y, particularmente, las matemáticas (Stelzer y Cervigni, 2011).

En estudiantes universitarios la información es contradictoria. Barceló, Lewis y Moreno (2006) no fueron capaces de encontrar relaciones entre las diferentes tareas del Test de Clasificación de Cartas de Winsconsin, el Test de Stroop y el Test de Fluidez Verbal (fonológico), con el promedio académico de sus participantes (36 estudiantes de ingeniería, de entre 18 y 23 años de edad); aunque si se observó que los antecedentes familiares, psicológicos y académicos contribuían a las diferencias entre los estudiantes de bajo y alto rendimiento. Vergara (2011) tampoco encontró relaciones entre las FE y el RA (promedio académico) de sus participantes, cuyas edades iban de los 17 a los 25 años y se encontraban estudiando el primer año de psicología. Como FE se incluyeron a la Memoria de Trabajo, la Planeación, la Flexibilidad Mental, la Atención y la Inhibición; como medidas de funcionamiento ejecutivo se utilizaron el Test de Fluidez de Diseños de Ruff, el Trail Making Test, el Test de Clasificación de Tarjetas de Wisconsin, el Test de Palabras y Colores de Stroop y el Test de Fluidez Verbal. 


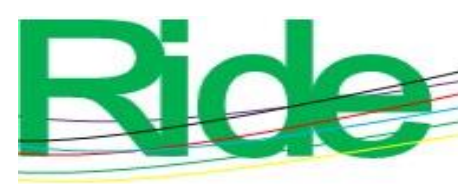

Revista lberoamericana para la

Investigación y el Desarrollo Educativo

ISSN 2007 - 7467

Finalmente tenemos el trabajo de Casas (2013), que investigó el funcionamiento ejecutivo (flexibilidad cognitiva, control inhibitorio, resistencia a la interferencia, memoria de trabajo y planificación) de 30 adultos de entre 18 y 25 años. Para ello se utilizó el Trail Making Test, el Test de Palabras y Colores de Stroop, el Mapa del ZOO, y la subprueba de memoria de trabajo del WAIS-III. En el estudio se encontraron relaciones significativas entre el control inhibitorio, la memoria de trabajo, y la planeación con el RA (promedio escolar).

En síntesis, aunque la investigación es clara sobre la existencia de relaciones entre FE con distintas formas de RA en la niñez, aún no es claro si esto se mantiene en edades posteriores. A este respecto, Best, Miller y Naglieri (2011) encontraron que, en una muestra de 2036 sujetos de entre 5 y 17 años, a los que se evaluó con el Cognitive Assessment System (CAS), el desempeño en las tareas de FE (Matching Numbers, Planned Codes, Planned Connections y la puntuación total del CAS) mejoró hasta la edad de 15 años y entonces dicha mejora disminuyó, con patrones distintos entre las diferentes tareas utilizadas. En su caso la medida de RA fue el Woodcock-Johnson Tests of Achievement-Revised, y no encontraron diferencias reales entre el área de matemáticas y lectura. Entre sus resultados más importantes fue: que la relación entre el tiempo y la exactitud para realizar las tareas, dependía de la tarea y de la edad de la persona.

De tal manera, aún se requieren más investigaciones en el área y, particularmente en edades intermedias a los estudios realizados hasta el momento; sobre todo por los cambios durante la adolescencia que en el desarrollo de los lóbulos frontales se han descrito en la literatura (Casey, et al, 2011, Kuhn 2006, Rosselli, Jurado y Matute, 2008). En ese sentido parece haber consenso sobre un desarrollo secuencial de las FE a lo largo de la vida, que va de un desarrollo aislado entre sus componentes hasta su integración compleja, un desarrollo piramidal en las que las más básicas soportan el desarrollo de las más complejas, y un incremento lineal en la infancia que tiene su meseta en la adolescencia (Flores-Lázaro, Castillo-Preciado y Jiménez-Miramonte, 2014).

En tal sentido, Flores-Lázaro, et al. (2014) expresa: 


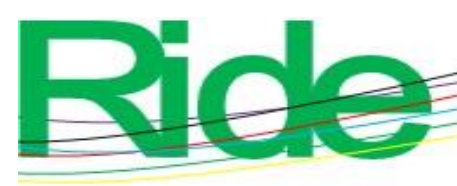

Revista Iberoamericana para la

Investigación y el Desarrollo Educativo

ISSN 2007 - 7467

La revisión de la literatura indica que en la infancia temprana se desarrollan la detección de selecciones de riesgo y el control inhibitorio; en la infancia tardía la memoria de trabajo, la flexibilidad mental, la memorización estratégica, y la planeación visoespacial; mientras que en la adolescencia tardía se terminan de desarrollar la planeación secuencial. En la juventud continúan su desarrollo la fluidez verbal y la abstracción, pero sólo en contextos escolarizados (p. 468).

Dada la información acumulada hasta el momento, debemos considerar que, independientemente del desarrollo diferencial de las FE a lo largo de la vida, teóricamente su importancia para el desempeño académico sigue manteniéndose, porque las FE permiten la planeación, regulación y verificación de la conducta, particularmente ante contextos novedosos, como los que se encuentran en las actividades escolares y que van siendo cada vez más exigentes conforme se avanza en el nivel educativo.

Las inconsistencias entre los hallazgos pueden deberse a varias situaciones. En primer lugar, a las diferencias metodológicas entre componentes de funcionamiento ejecutivo involucrados y las tareas específicas utilizadas para medirlas. Pero también, debido al tipo de población incluida. En el caso de los universitarios, es claro que para ese nivel ya ha habido una clara eliminación de una gran franja poblacional quedando los más aptos para contender con los estudios, lo cual podría significar un desempeño más homogéneo tanto en las FE como en el RA. La medida misma de RA sería otra de las diferencias a tomar en cuenta entre los estudios, pues, mientras que en las investigaciones con niños las medidas han sido diferenciadas, en los jóvenes y adultos generalmente solo se ha tomado el promedio general.

Con relación a las medidas de funcionamiento ejecutivo hay que considerar que, su estudio ha estado vinculado más expresamente con la neuropsicología, que ha logrado sus avances a partir de la investigación con personas con daño cerebral, por lo que muchas de las tareas de evaluación empleadas en ese ámbito buscan la detección de daño neurológico y, con ello, la distinción entre un desempeño normal y uno anormal es más tajante en comparación con otras medidas cognitivas desarrolladas desde otras disciplinas. Pese a ello, también es cierto que, los test más populares de funcionamiento ejecutivo, como el Wisconsin Card Sorting 


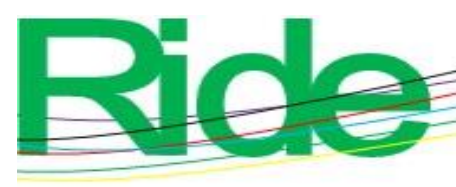

Revista Iberoamericana para la

Investigación y el Desarrollo Educativo

ISSN $2007-7467$

Test o el Trail Making Test, fueron creados en contextos ajenos a la neuropsicología (Marino y Julián, 2010).

Por otra parte, existe el problema de que en las investigaciones algunos autores utilizan un test completo como medida de un área específica, mientras que otros dividen al test en subtareas y las utilizan como medidas de habilidades distintas. Por ello, una solución a las dificultades metodológicas inherentes a la evaluación es el uso de medidas basadas en modelos neuropsicológicos del funcionamiento ejecutivo, como es el caso de la Batería Neuropsicológica de funciones ejecutivas (Flores, Ostrosky-Solís y Lozano, 2008) que basa su selección de las pruebas incluidos en ella, en un modelo de funcionamiento ejecutivo asociado a regiones anotomo-funcionales de la corteza frontal.

Con relación a la población de estudio, una opción a la posible homogeneidad de los grupos es contrastar los resultados por nivel de rendimiento. Esto se ha venido realizando, sin embargo, si la población de niveles educativos medios se ha homogenizado, las diferencias en los rendimientos serán menores con lo que la varianza muestral tenderá a serlo también y ello podría minimizar los efectos. Otra opción es contar con muestras de poblaciones marcadamente desaventajada con otras más favorecidas en términos de sus antecedentes de estimulación ambienta y/o condición socioeconómica.

Teniendo en cuenta todo ello, el presente estudio buscó averiguar (a) si existen relaciones entre las tareas y componentes del funcionamiento ejecutivo, medidas por la BANFE, con el rendimiento académico de los participantes, y (b) si existen diferencias en el rendimiento académico y/o el funcionamiento ejecutivo por condición de procedencia, y otras variables demográficas entre estudiantes de un bachillerato que tiene una población interna a una casa hogar y estudiantes externos al mismo. ${ }^{1}$

\footnotetext{
${ }^{11}$ Cabe mencionar que, en la revisión de la literatura llevada a cabo, no se pudo identificar ningún estudio de habla inglesa o española que hubiese investigado la relación entre las variables señaladas en el nivel bachillerato, con lo que el estudio propuesto sería el primero en realizarse.
} 


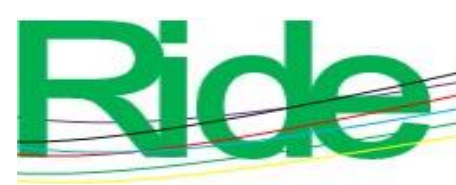

Revista Iberoamericana para la

Investigación y el Desarrollo Educativo

ISSN $2007-7467$

\section{Método}

Se realizó un estudio de campo de evaluación sistemática (Scott y Wertheimer, 1981) bajo una estrategia transversal simple (Baltes, Reese y Nesselroade, 1981) y con un diseño intragrupo (Bialystok, 1988), no experimental (Kerlinger y Lee, 2002).

\section{Participantes}

Se contó con la participación de 50 alumnos de primer semestre de bachillerato tecnológico, adscrito a una casa hogar. Por esta característica, el bachillerato cuenta con una población de estudiantes internos, provenientes de diferentes puntos de la república mexicana, aunque con mayor representación de los estados de Morelos y Guerrero; así como estudiantes externos del Estado de Morelos. Los estudiantes internos cuentan con historias de diferente grado de abandono, pobreza y marginación. Algunos han sido abandonados y otros, a pesar de que cuentan con padres o familiares cercanos no están en condiciones de pagar sus estudios por esta razón son internados en la casa hogar. La edad de ingreso a la institución varía desde la primera infancia y la adolescencia. Debido a estas circunstancias y a otras ligadas a la organización interna de la casa hogar, los estudiantes internos tienden a ingresar al bachillerato en edades posteriores y con un rango mayor de diferencia que los estudiantes externos.

La selección tanto de la población como de la muestra se hizo de forma no probabilística y fue de tipo accidental (Kerlinger y Lee, 2002). Para reunir la muestra se les explicaron los objetivos de la investigación a todos los estudiantes del primer semestre y se conformó el grupo de participantes con aquellos que voluntariamente decidieron hacerlo y cumplieron con los criterios de inclusión. Para obtener la autorización se les otorgó una carta consentimiento dirigida a los padres de familia de los alumnos externos y a la autoridad tutelar de los internos.

El bachillerato ofrece programas de estudio semestrales a lo largo de 3 años, brindando las especialidades de contabilidad, electrónica, puericultura y turismo, por lo que esta fue tomada como una variable de investigación, lo mismo que el género y la edad. La población final de 
estudio estuvo formada como se muestra en la tabla 1. En total hubo 26 adolescentes externos y 24 adolescentes internos, con un rango de edad de entre 15 a 19 años.

Tabla 1. Características básicas de los estudiantes incluidos en la muestra.

\begin{tabular}{|l|l|l|l|l|l|}
\hline & \multicolumn{2}{|l|}{ Estudiantes } & \multicolumn{2}{l|}{ Estudiantes } & \\
\hline & Hombres & Mujeres & Hombres & Mujeres & Total \\
\hline Contabilidad & 2 & 4 & 1 & 3 & 10 \\
\hline Electrónica & 4 & 2 & 6 & 2 & 14 \\
\hline Puericultura & 0 & 9 & 1 & 5 & 15 \\
\hline Turismo & 2 & 1 & 2 & 6 & 11 \\
\hline Total & 8 & 16 & 10 & 16 & \\
\hline
\end{tabular}

Fuente: Elaboración propia

Los criterios de inclusión contemplaron las siguientes condiciones:

- Alumnos que pertenecieran al primer semestre de bachillerato

- En estatus académico regular.

- Concluyera con la aplicación de los instrumentos de estudio

\section{Instrumentos}

Se utilizó la Batería Neuropsicológica de Funciones Ejecutivas y Lóbulos Frontales (BANFE-2) (Flores, Ostrosky-Solís y Lozano, 2008). Dicho instrumento agrupa 15 pruebas neuropsicológicas que han mostrado una alta confiabilidad y validez en la evaluación de procesos cognitivos dependientes de la corteza prefrontal. Su diseño se basa en una división anatómo-funcional de la corteza frontal en cuatro áreas: corteza órbitofrontal, corteza prefrontal medial, corteza prefrontal dorsolateral y corteza prefrontal anterior. Las tareas que la conforman son de baja complejidad cognitiva y alta sensibilidad al daño de un área cerebral específica. Los procesos evaluados por la BANFE se presentan en la tabla 2 y están organizados según su mayor-menor complejidad.

La calificación de la batería se da en términos de dimensiones que involucran tareas de test diferentes. Así, un test como Laberintos tiene tareas que aportan información para la dimensión orbitomedial y otras para la dorsolateral. Otra característica de la BANFE, ligada a su calificación es que, no toda la información (puntuaciones diferenciadas) de cada subprueba es tomada en cuenta para la elaboración del perfil final. Por ello, para este estudio se tomaron en cuenta solo las puntuaciones que aportan información para el perfil de 
funcionamiento. Finalmente, la batería realiza una clasificación de las puntuaciones por dimensiones en niveles de desempeño; a saber: Normal Alto, Normal, Alteración LeveModerada y Alteración Severa. Estos niveles también fueron tomados en cuenta para el análisis.

Tabla 2. Mapa conceptual de la BANFE

\begin{tabular}{|c|c|}
\hline \multirow{3}{*}{$\begin{array}{l}\text { Metafunciones (Corteza Prefrontal } \\
\text { Anterior). }\end{array}$} & Metamemoria. \\
\hline & Comprensión de sentido figurado. \\
\hline & Actitud abstracta. \\
\hline \multirow{7}{*}{$\begin{array}{l}\text { Funciones Ejecutivas (Corteza } \\
\text { Prefrontal Dorsolateral. }\end{array}$} & Fluidez verbal. \\
\hline & Productividad. \\
\hline & Flexibilidad mental. \\
\hline & Planeación visoespacial. \\
\hline & Planeación secuencial. \\
\hline & Secuenciación inversa. \\
\hline & Control de codificación. \\
\hline \multirow{3}{*}{$\begin{array}{l}\text { Memoria de trabajo (corteza } \\
\text { Prefrontal Dorsolateral). }\end{array}$} & Memoria de trabajo visual autodirigida. \\
\hline & Memoria de trabajo verbal ordenamiento. \\
\hline & Memoria de trabajo visoespacial secuencial. \\
\hline \multirow{3}{*}{$\begin{array}{l}\text { Funciones básicas } \quad \text { (Corteza } \\
\text { orbitofrontal y Corteza Prefrontal } \\
\text { Medial). }\end{array}$} & Control inhibitorio. \\
\hline & Seguimiento de reglas. \\
\hline & Procesamiento riesgo-beneficio. \\
\hline
\end{tabular}

Fuente: Adaptado de Flores, Ostrosky-Solís y Lozano (2008, p. 145).

Las pruebas que integran la batería son: Efecto Stroop, Laberintos, Juego de Cartas, Señalamiento Autodirigido, Memoria de Trabajo Visoespacial, Ordenamiento Alfabético de Palabras, Clasificación de Cartas, Torre de Hanoi, Resta y Suma Consecutivas, Fluidez Verbal, Selección de Refranes, Clasificaciones Semánticas y Metamemoria.

\section{Rendimiento Académico}

El rendimiento académico se obtuvo por el promedio al finalizar el semestre de las materias de álgebra y de expresión oral y escrita, así como del promedio general de las materias de tronco común; que además de las dos anteriores incluían química, inglés, ciencia, tecnología, sociedad y valores y tecnología de la información y la comunicación.

\section{Procedimiento}

Se administró la BANFE a los participantes de forma individual por examinadores entrenados, en un salón del propio bachillerato, aislado del ruido, amplio y bien iluminado. 
Se siguieron los procedimientos de administración descritos por la batería y la aplicación se dio en una sola sesión.

\section{Análisis estadísticos}

Debido a que algunas de las variables analizadas eran de tipo categórico y, a que las pruebas Shapiro-Wilk mostraron que la mayoría de las variables continuas no se comportaron de manera normal, para el análisis de los datos se llevaron a cabo análisis no paramétricos. En primer lugar, se realizaron análisis de correlación de Spearman para identificar las relaciones significativas entre las variables demográficas (género, edad, espacialidad y grupo de procedencia [internos y externos]); de funcionamiento ejecutivo (dimensiones diagnósticas de la BANFE, y subpruebas del perfil diagnóstico); y de rendimiento académico (Tronco Común, Álgebra y Expresión Oral y Escrita). En segundo lugar, se llevaron a cabo pruebas de Kruskal-Wallis y/o Pruebas U de Mann-Whitney entre las variables que presentaron correlaciones significativas.

\section{Resultados}

\section{Relaciones y comparaciones generales}

En la tabla 3 se observa que se presentaron relaciones significativas entre varias de las dimensiones de FE con las variables de RA como con las demográficas; mientras que solo la procedencia de los participantes estuvo relacionada con la Expresión Oral y Escrita. Con ello el FE se muestra importante en la expresión de RA en general; a excepción de la dimensión Prefrontal Anterior que no mostró relación significativa alguna con ningún área del RA. Los resultados de las pruebas Kruskal-Wallis llevadas a cabo entre las variables que mostraron interacciones (ver tabla 4)., cuando cada variable de RA se dividió en niveles de rendimiento, tan sólo Álgebra mostró diferencias significativas entre los grupos. Las pruebas U de Mann-Whitney realizadas para indagar entre qué grupos se daban las diferencias, mostró que, en el caso de las FE orbitomediales el nivel de funcionamiento ejecutivo difirió entre los grupos de rendimiento bajo y medio $(z=-2.288, p<.05)$ y entre los grupos de 
rendimiento bajo y alto $(\mathrm{z}=-2.746, \mathrm{p}<.01)$. Para las FE dorsolaterales, las diferencias se dieron entre los grupos de rendimiento bajo y alto $(\mathrm{z}=-2.305, \mathrm{p}<.05)$ y entre los grupos de rendimiento medio y alto $(\mathrm{z}=-2.396, \mathrm{p}<.05)$. Lo mismo sucedió en el caso de la puntuación global en la BANFE $(z=-2.805, p<.01 \mathrm{y} \mathrm{z}=-2.255, \mathrm{p}<.05$, respectivamente $)$.

Tabla 3. Coeficientes de correlación de Spearman entre las variables demográficas, las de Rendimiento Académico y las dimensiones diagnósticas de la BANFE.

\begin{tabular}{|c|c|c|c|c|c|c|c|c|c|c|c|}
\hline & 1 & 2 & 3 & 4 & 5 & 6 & 7 & 8 & 9 & 10 & 11 \\
\hline Media & -- & -- & -- & -- & 7.92 & 7.78 & 8.44 & 192.82 & 18.40 & 172.20 & 383.22 \\
\hline $\mathrm{DE}$ & -- & -- & -- & -- & 1.16 & 1.52 & 1.57 & 6.88 & 3.51 & 24.56 & 27.75 \\
\hline 1. G & 1.00 & & & & & & & & & & \\
\hline 2. $\mathrm{E}$ & .02 & 1.00 & & & & & & & & & \\
\hline 3. Esp & -.17 & -.15 & 1.00 & & & & & & & & \\
\hline 4. $\mathrm{P}$ & .05 & $-.69^{* * *}$ & .15 & 1.00 & & & & & & & \\
\hline 5. TC & -.21 & -.03 & -.21 & .25 & 1.00 & & & & & & \\
\hline 6. A & -.03 & -.11 & -.18 & .16 & $.80^{* *}$ & 1.00 & & & & & \\
\hline 7. EOE & -.15 & -.03 & -.17 & $.28 *$ & $.87^{* *}$ & $.60^{* *}$ & 1.00 & & & & \\
\hline 8. OM & -.07 & -.03 & $-.30^{*}$ & -.01 & $.31^{*}$ & $.39^{* * *}$ & .19 & 1.00 & & & \\
\hline 9. PA & -.19 & $.28^{*}$ & -.01 & -.17 & .03 & -.07 & -.04 & -.01 & 1.00 & & \\
\hline 10. DL1 y 2 & $.35^{*}$ & -.16 & -.27 & $.29^{*}$ & $.33^{*}$ & $.3^{*}$ & $.29^{*}$ & .25 & .17 & 1.00 & \\
\hline 11. BANFE & .27 & -.12 & $-.31^{*}$ & .26 & $.36^{* *}$ & $.38^{* *}$ & $.30^{*}$ & $.41^{* *}$ & .26 & $.97^{* * *}$ & 1.00 \\
\hline
\end{tabular}

G: Género; E: Edad; Esp: Especialidad; P: Procedencia; TC: Tronco Común; A: Álgebra; EOE: Expresión Oral y Escrita; OM: Orbitomedial; PA: Prefrontal anterior; DL1 y 2: Dorsolateral Memoria de Trabajo + Funciones Ejecutivas; BANFE: resultado total.

* La correlación es significativa en el nivel 0,05 ( 2 colas).

**. La correlación es significativa en el nivel 0,01 (2 colas).

Fuente: Elaboración propia

Tabla 4. Comparaciones de los resultados en las FE para los diferentes niveles de RA, mediante la Prueba de Kruskal-Wallis.

\begin{tabular}{|c|c|c|c|c|c|c|c|c|c|c|}
\hline & & \multicolumn{3}{|c|}{ Tronco Común } & \multicolumn{3}{|c|}{ Álgebra } & \multicolumn{3}{|c|}{ Expresión Oral y Escrita } \\
\hline $\mathrm{FE}$ & Niveles de RA & $\mathrm{N}$ & $\mathrm{R}$ & $\mathrm{X}^{2^{*}}$ & $\mathrm{~N}$ & $\mathrm{R}$ & $\mathrm{X}^{2^{*}}$ & $\mathrm{~N}$ & $\mathrm{R}$ & $\mathrm{X}^{2^{*}}$ \\
\hline \multirow{3}{*}{$\mathrm{OM}$} & Bajo & 9 & 23.67 & \multirow{3}{*}{3.462} & 9 & 13.78 & & 7 & 19.71 & \multirow{3}{*}{1.313} \\
\hline & Medio & 12 & 19.50 & & 28 & 26.61 & $8.028 * *$ & 12 & 25.88 & \\
\hline & Alto & 29 & 28.55 & & 13 & 31.23 & & 31 & 26.66 & \\
\hline \multirow{3}{*}{ DL1 y 2} & Bajo & 9 & 20.17 & \multirow{3}{*}{1.572} & 9 & 22.61 & & 7 & 22.29 & \multirow{3}{*}{1.301} \\
\hline & Medio & 12 & 25.54 & & 28 & 22.14 & $7.057 * *$ & 12 & 22.63 & \\
\hline & Alto & 29 & 27.14 & & 13 & 34.73 & & 31 & 27.34 & \\
\hline \multirow{3}{*}{ BANFE } & Bajo & 9 & 20.17 & \multirow{3}{*}{1.818} & 9 & 19.17 & & 7 & 22.43 & \multirow{3}{*}{1.463} \\
\hline & Medio & 12 & 24.58 & & 28 & 23.16 & $7.855^{* *}$ & 12 & 22.25 & \\
\hline & Alto & 29 & 27.53 & & 13 & 34.92 & & 31 & 27.45 & \\
\hline
\end{tabular}

$* 2 \mathrm{gl}$.

$* * \mathrm{P}<.05$

Fuente: Elaboración propia 


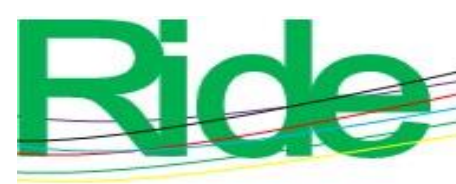

Revista Iberoamericana para la

Investigación y el Desarrollo Educativo

ISSN 2007 - 7467

En la Tabla 5 se observan los resultados de U de Mann-Whitney para la dimensión dorsolateral y para el RE en Expresión Oral y Escrita por las variables demográficas de género y procedencia. En todos los casos se observaron diferencias estadísticamente significativas entre los grupos. Cabe señalar que una correlación que también resultó significativa fue la especialidad de estudio, por una parte, y las FE orbitomediales y la puntuación total de la BANFE, por la otra; sin embargo, al llevarse a cabo las pruebas estadísticas, no se pudo demostrar diferencias significativas entre las diferentes interacciones.

Tabla 5. Comparaciones de los resultados en las FE para las diferentes categorías de

Género y Condición de Procedencia.

\begin{tabular}{llrrr}
\hline & Género & $\mathrm{N}$ & $\mathrm{R}$ & $\mathrm{Z}$ \\
\hline \multirow{2}{*}{ DL1 y 2 } & Mujer & 32 & 21.69 & $-2.467^{*}$ \\
& Hombre & 18 & 32.28 & \\
\hline \multirow{2}{*}{ DL1 y 2 } & Interno & 24 & 21.21 & $-2.001^{*}$ \\
& Externo & 26 & 29.46 & \\
\hline \multirow{2}{*}{ EOE } & Interno & 21.46 & 21.45 & $-1.969^{*}$ \\
& Externo & 29.23 & 29.23 & \\
\hline R: rango promedio & & & \\
*P<.05 & & & &
\end{tabular}

Fuente: Elaboración propia

\section{Relaciones y comparaciones específicas entre las tareas orbitomediales y el RA}

Como se puede ver en la tabla 6 la mayoría de las correlaciones que resultaron significativas los fueron con el promedio de Álgebra, seguida de Tronco Común y solo una con Expresión Oral y Escrita. También se debe hacer notar que el tiempo y los Errores Tipo Stroop, que mostraron significancia, correlacionaron de forma inversa con el rendimiento académico, mientras que las otras tareas lo hicieron de forma directa; incluso para los errores de atravesar, en el caso de Laberintos. Este último dato es interesante porque parece indicar que aquellos que son menos controlados en el trazo en los Laberintos tienen un mejor promedio de tronco común. También debe señalarse que, aunque las tareas orbitomediales que componen la BANFE se asocian a las FE de Control Inhibitorio, Seguimiento de Reglas y Procesamiento Riesgo-Beneficio, todas las tareas que resultaron significativas están a asociadas al Control Inhibitorio y no hubo alguna que perteneciera a las otras áreas. 


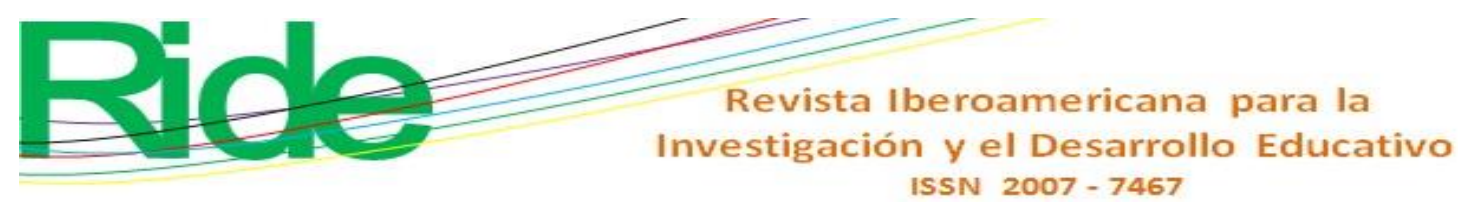

$\mathrm{Al}$ examinarse las relaciones intragrupo, de acuerdo con los niveles de rendimiento, se pudo observar que las veces que se atraviesa en Laberintos mostró diferencias intragrupales para el rendimiento en Tronco Común; que no hubo diferencias significativas en el caso de la Expresión Oral y Escrita y que fue la variable de Álgebra la que mostro más diferencias intragrupales. En la tabla 7 se presentan los resultados que fueron significativos.

Tabla $6^{2}$. Coeficientes de correlación de Spearman entre las variables de Rendimiento Académico y las Tareas Orbitomediales.

\begin{tabular}{|c|c|c|c|}
\hline & 1 & 2 & 3 \\
\hline Media & 7.92 & 7.78 & 8.44 \\
\hline$\overline{\mathrm{DE}}$ & 1.16 & 1.52 & 1.57 \\
\hline 1. Tronco Común & 1.000 & & \\
\hline 2. Álgebra & $.803^{* *}$ & 1.000 & \\
\hline 3. Expresión Oral y Escrita & $.872^{* *}$ & $.599 * *$ & 1.000 \\
\hline 4. Laber & $.375^{* * *}$ & .257 & .256 \\
\hline 5. Juego & -.047 & -.084 & .046 \\
\hline 6. Juego de Cartas: Porcentaje de Cartas de Riesgo & .073 & .169 & .108 \\
\hline 7. Stroop A & $.391^{* *}$ & .428 ** & $.327^{*}$ \\
\hline 8. Stroop A: Tiem & -.199 & $-.287^{*}$ & -.148 \\
\hline 9. Stroop A: Errores Tipo Stroop & -.201 & -.256 & -.145 \\
\hline 10. Stroop B: Aciertos & $.444^{* *}$ & $.480 * *$ & .273 \\
\hline 11. Stroop B: Tiempo & -.277 & $-.314^{*}$ & -.181 \\
\hline 12. Stroop B: Errores & $-.286^{*}$ & $-.300^{*}$ & -.158 \\
\hline 13. Clasificación de Cartas: Errores de Mantenimiento & .041 & .061 & .090 \\
\hline
\end{tabular}

*. La correlación es significativa en el nivel 0,05 ( 2 colas $)$.

**. La correlación es significativa en el nivel 0,01 (2 colas).

Fuente: Elaboración propia

\footnotetext{
${ }^{2}$ Para los fines del trabajo, no interesaba mostrar las relaciones entre las tareas de funcionamiento
} ejecutivo, la tabla de correlaciones se presenta recortada para facilitar su lectura. 


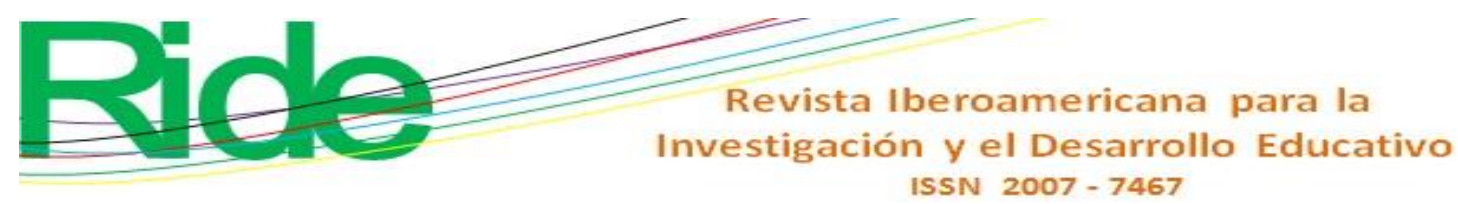

Tabla 7. Comparaciones de las tareas de FE orbitomediales para los diferentes niveles de RA, mediante la prueba de Kruskal-Wallis.

\begin{tabular}{llccc}
\hline & Categorías de RA & N & Rango promedio & $\mathrm{X}^{2^{*}}$ \\
\hline \multirow{4}{*}{ LA } & Tronco Común bajo & 9 & 24.17 & $8.895^{*}$ \\
& Tronco Común medio & 12 & 17.00 & \\
& Tronco Común alto & 29 & 29.43 & \\
\hline \multirow{4}{*}{ SA-A } & Álgebra bajo & 9 & 13.56 & $8.778^{*}$ \\
& Álgebra medio & 28 & 26.63 & \\
& Álgebra alto & 13 & 31.35 & \\
\hline \multirow{4}{*}{ SB-A } & Álgebra bajo & 9 & 15.89 & $11.912^{* *}$ \\
& Álgebra medio & 28 & 23.82 & \\
& Álgebra alto & 13 & 35.77 & \\
\hline \multirow{3}{*}{ SB-ETS } & Álgebra bajo & 8 & 31.44 & $6.011^{* *}$ \\
& Álgebra medio & 28 & 26.34 & \\
& Álgebra alto & 13 & 18.15 & \\
$*$ * $<.05$ & & & & \\
& & & & \\
& & &
\end{tabular}

Fuente: Elaboración propia

Las pruebas U de Mann-Whitney realizadas para indagar entre qué grupos se daban las diferencias, mostró que, no todas las relaciones que se mostraron significativas tuvieron diferencias intergrupales por nivel de rendimiento; excepto la de Laberintos Atraviesa y algunas de las asociadas a las tareas tipo Stroop. En el caso de Laberintos Atraviesa, hubo diferencias entre los grupos de rendimiento en Tronco Común bajo y medio $(z=-2.108, p$ $<.05)$ y entre los grupos de rendimiento en Tronco Común medio y alto $(\mathrm{z}=-2.859, \mathrm{p}<.05)$. Para Aciertos en Stroop A, las diferencias se dieron, en el caso de Álgebra, entre los grupos de rendimiento académico bajo y medio $(\mathrm{z}=-2.235, \mathrm{p}<.05)$ y entre los grupos de rendimiento bajo y alto $(\mathrm{z}=-3.133, \mathrm{p}<.01)$. En el caso de Aciertos Stroop B, las diferencias se encontraron entre los grupos de rendimiento bajo y alto $(z=-3.221, p<.01)$; y entre los grupos de rendimiento medio y alto. $(\mathrm{z}=-2.712, \mathrm{p}<.01)$. Esto mismo sucedió con la tarea de Errores Tipo Stroop B $(z=-2.245, p<.05$ y z $=-2.015, p<.05)$ 


\section{Relaciones y comparaciones específicas entre las tareas dorsolaterales (Memoria de} Trabajo) y el RA

La tabla 8 muestra las correlaciones significativas entre las tareas dorsolaterales de memoria de trabajo y las de RA. En ella se puede observar que hubo más relaciones entre el RA en Expresión Oral y Escrita que en las otras dos variables de rendimiento; y que los aciertos en la tarea de Resta Consecutiva B mostraron relación con las tres áreas de funcionamiento. Es interesante notar que la tarea que involucra tiempo se relaciona inversamente con las áreas de rendimiento de Álgebra y Expresión Oral y Escrita, mientras que las otras tareas lo están directamente, a pesar de que algunas representan desempeños erróneos; por ejemplo, las perseveraciones en el señalamiento autodirigido por lo que, a medida que aumentan, también lo hace el rendimiento de Álgebra y Tronco Común. También debe señalarse que, aunque las tareas dorsolaterales que componen la BANFE se asocian a las FE de Memoria de Trabajo de tipo visoespacial secuencial, autodirigida y verbal de ordenamiento, las tareas que resultaron significativas se asocian a las dos primeras solamente y no hubo asociación con tareas de memoria verbal de ordenamiento.

Las pruebas Kruskal-Wallis realizadas para observar las posibles diferencias intragrupales entre las variables con correlaciones significativas mostraron significatividad en el caso del RA en Álgebra. Los resultados para dicha variable se muestran en la tabla 9. Por su parte, las pruebas U de Mann-Whitney señalaron como significativas las diferencias entre los grupos de rendimiento bajo y medio $(\mathrm{z}=-2.579 ; \mathrm{p}=.01)$ para las perseveraciones en Señalamiento Autodirigido. 


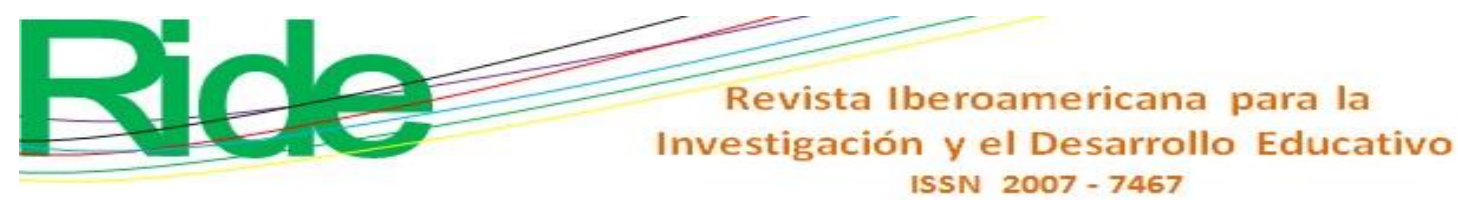

Tabla 8. ${ }^{3}$ Coeficientes de correlación de Spearman entre las variables de Rendimiento Académico y las Tareas Dorsolaterales: Memoria de Trabajo.

\begin{tabular}{|c|c|c|c|}
\hline & 1 & 2 & 3 \\
\hline Media & 7.78 & 8.44 & 7.92 \\
\hline$\overline{\mathrm{DE}}$ & 1.52 & 1.57 & 1.16 \\
\hline 1. Álgebra & 1.000 & & \\
\hline 2. Expresión Oral y Escrita & $.599 * *$ & 1.000 & \\
\hline 3. Tronco Común & $.803^{* * *}$ & $.872^{* * *}$ & 1.000 \\
\hline $\begin{array}{l}\text { 4. Señalamiento Autodirigido: } \\
\text { Perseveraciones }\end{array}$ & $.284^{*}$ & .194 & $.351^{*}$ \\
\hline 5. Señalamiento Autodirigido: Tiempo & -.134 & -.105 & -.095 \\
\hline 6. Señalamiento Autodirigido: Aciertos & .276 & .132 & .191 \\
\hline 7. Resta Consecutiva "A": Tiempo & -.211 & $-.309^{*}$ & $-.327^{*}$ \\
\hline 8. Resta Consecutiva "A": Aciertos & .181 & .148 & .158 \\
\hline 9. Resta Consecutiva "B": Tiempo & -.096 & -.217 & -.201 \\
\hline 10. Resta Consecutiva "B": Aciertos & $.404^{* *}$ & $.435^{* *}$ & $.387^{* *}$ \\
\hline 11. Suma Consecutiva: Tiempo & -.207 & -.145 & -.115 \\
\hline 12. Suma Consecutiva: Aciertos & .081 & .171 & .084 \\
\hline 13. Ordenamiento Alfabético 1 & -.114 & -.096 & -.123 \\
\hline 14. Ordenamiento Alfabético 2 & -.021 & -.158 & -.133 \\
\hline 15. Ordenamiento Alfabético 3 & -.071 & -.257 & -.148 \\
\hline $\begin{array}{l}\text { 16. Memoria Visoespacial: Secuencia } \\
\text { Máxima }\end{array}$ & .021 & -.019 & -.056 \\
\hline 17. Memoria Visoespacial: Perseveraciones & .196 & -.013 & .001 \\
\hline 18. Memoria Visoespacial: Errores de Orden & -.135 & .068 & -.005 \\
\hline
\end{tabular}

Fuente: Elaboración propia

Tabla 9. Comparaciones de las tareas de FE dorsolateral (Memoria de Trabajo) para los diferentes niveles de RA, mediante la Prueba de Kruskal-Wallis

\begin{tabular}{llccc}
\hline & Rango de Álgebra & N & Rango promedio & $\mathrm{X}^{2^{*}}$ \\
\hline \multirow{3}{*}{ SA-P } & Rendimiento algebra bajo & 9 & 14.83 & \multirow{2}{*}{$6.286^{*}$} \\
& Rendimiento algebra medio & 28 & 27.00 & \\
& Rendimiento algebra alto & 13 & 29.65 & \\
\hline \multirow{3}{*}{ RCB-A } & Rendimiento algebra bajo & 9 & 20.00 & \multirow{2}{*}{$7.055^{*}$} \\
& Rendimiento algebra medio & 28 & 23.09 & \\
& Rendimiento algebra alto & 13 & 34.50 & \\
\hline
\end{tabular}

$* \overline{\mathrm{P}}<.05$

Fuente: Elaboración propia

\footnotetext{
${ }^{3}$ Para los fines del trabajo, no interesaba mostrar las relaciones entre las tareas de funcionamiento ejecutivo, la tabla de correlaciones se presenta recortada para facilitar su lectura.
} 


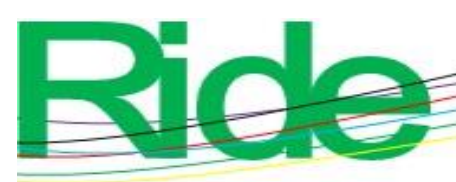

Revista Iberoamericana para la

Investigación y el Desarrollo Educativo

ISSN $2007-7467$

\section{Relaciones y comparaciones específicas entre las tareas dorsolaterales (Funciones Ejecutivas) y el RA}

Como se muestra en la tabla 10 tanto las tareas de Clasificación de Cartas: Aciertos y FV-P Fluidez Verbal: Perseveraciones se relacionaron significativamente con las tres variables de RA. Fluidez Verbal: Aciertos, lo hizo con Tronco Común y Álgebra, y Clasificación Semántica: Promedio Total, únicamente se relacionó con Álgebra. Todas las relaciones fueron directamente proporcionales a pesar de que pudiera tratarse de un desempeño considerado erróneo; que es el caso de FV-P Fluidez Verbal: Perseveraciones. También debe señalarse que, aunque las tareas dorsolaterales de Funciones Ejecutivas que componen la BANFE se asocian a las FE de Fluidez Verbal, Productividad, Flexibilidad Mental, Planeación visoespacial, Planeación Secuencial, Secuenciación Inversa y Control de Codificación, todas las tareas que resultaron significativas se asocian a la función de Flexibilidad Mental y no hubo alguna que perteneciera a las otras áreas.

Al llevarse a cabo las pruebas de comparación mediante Kruskal-Wallis, se encontró que Álgebra es la variable que más tiene diferencias intragrupales, mientras que las otras dos variables de rendimiento tuvieron diferencias intragrupales para una sola de las relaciones estudiadas (tabla 11). No se pudieron llevar a cabo las pruebas U de Mann-Whitney para la relación encontrada entre Fluidez Verbal: Perseveraciones y Tronco Común, por no haber suficientes casos válidos. En cuanto a Álgebra las pruebas mostraron diferencias entre los grupos de rendimiento bajo y alto para Clasificación de Cartas: Aciertos $(\mathrm{z}=-2.173 ; \mathrm{p}<.05)$; Clasificación Semántica: Promedio Total ( $\mathrm{z}=-2.182 ; \mathrm{p}<.05)$; Fluidez Verbal: Aciertos ( $\mathrm{z}=$ $--2.455 ; \mathrm{p}<.05)$; y Fluidez Verbal: Perseveraciones $(\mathrm{z}=-2.667 ; \mathrm{p}<.05)$. También hubo diferencias entre los grupos de rendimiento medio y alto en cuestión a Álgebra respecto a las tareas de Clasificación de Cartas: Aciertos ( $\mathrm{z}=-2.329 ; \mathrm{p}<.05)$; Clasificación Semántica: Promedio Total $(\mathrm{z}=-2.190 ; \mathrm{p}<.05)$. Para el rendimiento académico en Expresión Oral y Escrita, con relación a la tarea de Fluidez Verbal: Perseveraciones, las diferencias se dieron entre los grupos de rendimiento bajo y alto $(z=-2.701 ; p<.01)$ y entre los de rendimiento medio y alto $(\mathrm{z}=-1.992 ; \mathrm{p}<.05)$. 


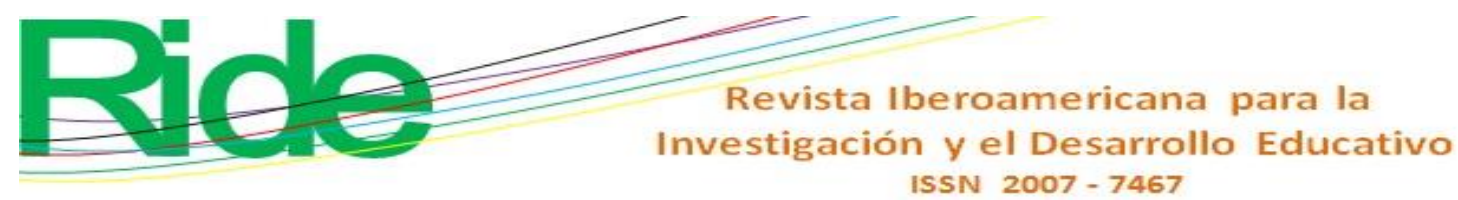

Tabla 10. ${ }^{4}$ Coeficientes de correlación de Spearman entre las variables de Rendimiento Académico y las Tareas Dorsolaterales: Funciones Ejecutivas.

\begin{tabular}{|c|c|c|c|}
\hline & 1 & 2 & 3 \\
\hline Media & 7.78 & 8.44 & 7.92 \\
\hline$\overline{\mathrm{DE}}$ & 1.52 & 1.57 & 1.16 \\
\hline 1. Álgebra & 1.000 & & \\
\hline 2. Expresión Oral & .599 ** & 1.000 & \\
\hline 3. Tronco Común & $.803^{* *}$ & $.872^{* *}$ & 1.000 \\
\hline 4. Laberintos: Planeación & .171 & -.047 & .124 \\
\hline 5. Laberintos: Tiempo & -.047 & -.116 & -.091 \\
\hline 6. Clasificación de Cartas: Aciertos & $.389^{* *}$ & $.329^{*}$ & $.3966^{* *}$ \\
\hline 7. Clasificación de Cartas: Perseveraciones & -.258 & -.187 & -.224 \\
\hline $\begin{array}{l}\text { 8. Clasificación de Cartas: Perseveraciones } \\
\text { Diferidas }\end{array}$ & -.202 & -.095 & -.182 \\
\hline 9. Clasificación de Cartas: Tiempo & .079 & .065 & .025 \\
\hline 10. Clasificación Semántica: Total de Categorías & .036 & -.082 & -.018 \\
\hline 11. Clasificación Semántica: Promedio Total & $.285^{*}$ & .070 & .079 \\
\hline 12. Clasificación Semántica: Puntuación Total & .046 & .004 & .014 \\
\hline 13. Fluidez Verbal: Aciertos & $.361^{*}$ & 216 & $.315^{*}$ \\
\hline 14. Fluidez Verbal: Perseveraciones & $.449^{* *}$ & $.344^{*}$ & $.423^{* *}$ \\
\hline 15. Torre de Hanoi 3 Discos: Movimientos & .056 & 125 & .112 \\
\hline 16. Torre de Hanoi 3 Discos: Tiempo & -.100 & .059 & .019 \\
\hline 17. Torre de Hanoi 4 Discos: Movimientos & -.120 & -.244 & -.225 \\
\hline 18. Torre de Hanoi 4 Discos: Tiempo & -.223 & -.181 & -.257 \\
\hline
\end{tabular}

*. La correlación es significativa en el nivel 0,05 (2 colas).

**. La correlación es significativa en el nivel 0,01 ( 2 colas).

Fuente: Elaboración propia

\section{Otras comparaciones}

Se decidió llevar a cabo comparaciones no paramétricas para establecer si los niveles diagnósticos de la BANFE (de alteración severa hasta normal alto) eran distintos para cada categoría de procedencia (usando U de Mann-Whitney); y para determinar si los rendimientos promedios en las variables de rendimiento académico eran diferentes según los niveles diagnósticos de la BANFE (usando Kruskal-Wallis). En ningún de los dos casos se observaron diferencias estadísticamente significativas, por lo que el diagnóstico dado a los estudiantes no parece ser diferente según su procedencia, ni parecen diferenciar entre los promedios obtenidos en las diferentes áreas de rendimiento académico.

\footnotetext{
${ }^{4}$ Paralos fines del trabajo, no interesaba mostrar las relaciones entre las tareas de funcionamiento ejecutivo, la tabla de correlaciones se presenta recortada para facilitar su lectura.
} 


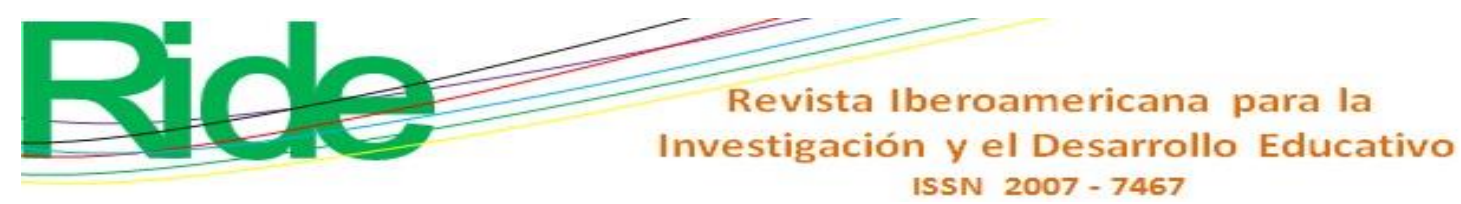

\section{Discusión}

A partir del análisis de los procesos asociados a las tareas específicas de cada prueba de la BANFE con el RA, se pudo constatar que las FE que mostraron asociación con el RA son el control inhibitorio, la memoria de trabajo secuencial y visoespacial, y la flexibilidad mental. Esto concuerda con los trabajos que han demostrado este tipo de asociaciones en edades tempranas ((Bryce, et al., 2015; Stelzer y Cervigni, 2011) y, parcialmente con el trabajo de Casas (2013), al coincidir en las asociaciones acerca del control inhibitorio y la memoria de trabajo, pero no en la planeación (resultado suyo) ni en la flexibilidad mental (resultado de este trabajo). Los resultados concuerdan con la literatura que muestra diferencias sobre distintos tipos de mediciones del RA. En el caso presente, las relaciones fueron más fuertes y consistentes en el caso del promedio en Álgebra lo cual también es consistente con los estudios previos (Stelzer y Cervigni, 2011).

Tabla 11. Comparaciones de las tareas de FE dorsolateral (Funciones Ejecutivas) para los diferentes niveles de RA, mediante la Prueba de Kruskal-Wallis

\begin{tabular}{llccc}
\hline & Niveles de RA & $\mathrm{N}$ & $\mathrm{R}$ & $\mathrm{X}^{2}$ \\
\hline \multirow{3}{*}{ FV-P } & Tronco Común bajo & 9 & 17.56 & \\
& Tronco Común medio & 12 & 20.67 & $8.250^{*}$ \\
& Tronco Común alto & 29 & 29.97 & \\
\hline \multirow{4}{*}{ CC-A A Álgebra bajo } & 9 & 21.33 & \\
& Álgebra medio & 28 & 22.71 & $6.604^{*}$ \\
& Álgebra alto & 13 & 34.38 & \\
\hline \multirow{3}{*}{ CS-PT } & Álgebra bajo & 9 & 21.50 & \\
& Álgebra medio & 28 & 22.96 & $6.061^{*}$ \\
& Álgebra alto & 13 & 33.73 & \\
\hline \multirow{3}{*}{ FV-A } & Álgebra bajo & 9 & 16.28 & \\
& Álgebra medio & 28 & 24.89 & $7.301^{*}$ \\
& Álgebra alto & 13 & 33.19 & \\
\hline \multirow{3}{*}{ FV-P } & Álgebra bajo & 9 & 17.56 & \\
& Álgebra medio & 28 & 24.50 & $7.851^{*}$ \\
& Álgebra alto & 13 & 33.15 & \\
\hline \multirow{3}{*}{ FV-P } & Expresión Oral y Escrita bajo & 7 & 14.50 & \\
& Expresión Oral y Escrita medio & 12 & 20.67 & $9.919 * *$ \\
& Expresión Oral y Escrita alto & 31 & 29.85 & \\
\hline *P<.05 & & & & \\
$* *$ P<.01 & & & &
\end{tabular}

Fuente: Elaboración propia 


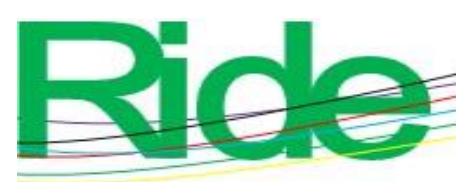

Revista Iberoamericana para la

Investigación y el Desarrollo Educativo

ISSN 2007 - 7467

Además, se pudo demostrar que la división de los estudiantes por grupos de rendimiento permitió constatar aprovechamientos distintos entre ellos, teniendo mejores puntuaciones los de rendimientos altos en comparación con los de rendimiento medio y bajo. Lo que no pudo demostrarse es que las dimensiones de clasificación clínica de la BANFE distinguieran entre el RA de los estudiantes. Esto parece significar que rendimientos neuropsicológicos anormales en las FE, incluso severos, no necesariamente se asocian con un RA consecuente; pero sí que, un rendimiento académico bajo, particularmente en Álgebra, se asocia más probablemente con menores rendimientos en las FE. Este es un resultado novedoso y podría implicar mecanismos de compensación entre los alumnos ante sus dificultades neuropsicológicas que, finalmente, les permiten contender con sus estudios de forma normal; pero que ante evaluaciones específicas se manifiestan en las áreas señaladas por la literatura. El hecho de que el promedio en Tronco Común y en Expresión Oral y Escrita, tuvieran menos asociaciones con las FE y menos diferencias intragrupo, es señal de que la relación entre las FE y el RA es diferenciada, y que puede haber interacciones internas entre estas variables que no se exploraron en este estudio. Además, debe señalarse el problema de la asignación de calificaciones entre cada materia, lo cual conlleva el problema de la subjetividad o parcialidad de las mismas, y que no pudo controlarse en este trabajo, por lo que estudios posteriores deberían medir el RA con evaluaciones objetivas y estandarizadas independientes de las calificaciones escolares, a la manera de Best, et al. (2011). Los resultados de este trabajo también muestran que usar el promedio global como indicador de RA podría estar escondiendo algunas relaciones específicas entre el $\mathrm{FE}$ con áreas específicas de funcionamiento académico, por lo que se recomienda tener medidas de RA por áreas de conocimiento, como se ha hecho en la investigación de infantes.

Con relación a las tareas específicas que mostraron relaciones consistentes con las comparaciones intragrupales, un resultado sorpresivo es el de que las perseveraciones en Fluidez Verbal y en Señalamiento Autodirigido se asociaron a mejores RA, toda vez que se trata de un dato de posible trastorno en la función ejecutiva (Sandson y Albert, 1984). Como las perseveraciones pueden presentarse en diferentes niveles de patología y tener diferentes mecanismos fisiopatológicos (Goldberg, 1986), no es claro el origen ni el significado de este resultado y debería ser motivo de estudio aparte. 


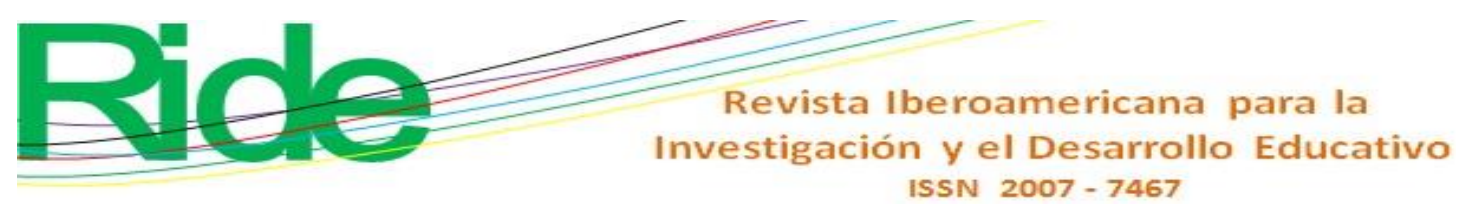

Con relación a las variables demográficas, aunque la especialidad de estudio, el género y la condición de procedencia, mostraron asociaciones con algunas dimensiones de funcionamiento ejecutivo, no se pudo constatar asociaciones globales con el rendimiento académico. Lo único que se observó es que las tareas de memoria de trabajo visoespacial y autodirigida mostraron diferencias por género y procedencia; con lo que podrían estar jugando un papel mediador en tales tareas dorsolaterales. También hay que señalar que, en el caso de la condición de procedencia, no se tuvo una medida específica de las diferencias entre los estudiantes internos en cuanto a sus antecedentes de estimulación, abandono y maltrato, que podrían ser mejores indicadores de funcionamiento ejecutivo que la mera distinción interno-externo. Esto debería tomarse en cuenta en otras investigaciones que busquen análisis multivariados y no sólo asociaciones entre dos variables (RA y FE en este caso).

Actualmente se sabe que el RA depende de variables sociales, institucionales y personales y, por ejemplo, Caso-Niebla y Hernández-Guzmán (2010) han elaborado un modelo explicativo del RA a partir de diferentes variables de esa naturaleza, lo cierto es que no han incluido las FE dentro de su modelo. A la luz de los hallazgos presentes y de lo encontrado previamente sería recomendable llevar a cabo trabajos de esa naturaleza; es decir que también incluyan a las FE como parte de un modelo explicativo. Una aproximación tipológica, centrada en personas sería especialmente provechosa por su capacidad para distinguir entre tipos de estudiantes y por ser adecuada para el trabajo con variables categóricas y continuas; además de ocuparse con interacciones entre variables de diferentes niveles y ser resistente al artefacto de linealidad en la investigación (Magnusson, 1998). 


\section{Conclusión}

El estudio de las relaciones entre las FE y el RA, particularmente el nivel medio superior, aún dista mucho de estar agotado. Este estudio está en la línea de aquellos que han mostrado relaciones significativas entre algunos componentes de las FE y medidas distintas del RA, no obstante, debe considerarse que los resultados no provienen de una muestra seleccionada al azar y están restringidos a una población específica que, por sus características institucionales, es distinta de otras poblaciones de bachilleres. Los resultados, con ello son difícilmente generalizables al resto de los estudiantes del nivel medio superior. Pese a ello, por el hecho de coincidir con la literatura científica, sobre que las FE de Control Inhibitorio, Memoria de Trabajo y Flexibilidad Mental se asocian al RA en edades inferiores, constituye un aporte significativo, porque permite continuar investigando el tema en un nivel educativo en el que no se ha realizado investigación al respecto, y del que el presente parece ser la primera experiencia en el área. El estudio además realiza aportes significativos sobre la asociación específica entre las variables estudiadas, y sobre la necesidad de incluir medidas diferenciadas del RA. Por ello, es necesario continuar investigando el tema, tanto a nivel restringido, y con otras poblaciones del mismo nivel educativo, como en estudios que incluyan otras variables sociales e institucionales que permitan entender de manera holística el RA; para ello se sugiere utilizar una aproximación centrada en personas, como lo sugiere la ciencia del desarrollo (Magnusson, 1998). 


\section{Referencias}

Anderson, V., Anderson, P., Northam, E., Jacobs, R. y Catroppa, C. (2001). Development of Executive Functions Through Late Childhood and Adolescence in an Australian Sample. Developmental Neuropsychology, 20(1), 385-406. doi: 10.1207/S15326942DN2001_5

Baltes, P. B., Reese, H. W. y Nesselroade, J. R. (1981). Métodos de investigación en psicología evolutiva: enfoque del ciclo vital. Madrid, España: Morata.

Barceló, E., Lewis, S. y Moreno, M. (2006). Funciones ejecutivas en estudiantes universitarios que presentan bajo y alto rendimiento académico. Psicología desde el Caribe, 18, 109-138. Recuperado de https://www.redalyc.org/pdf/213/21301806.pdf

Best, J., Miller, P. y Naglieri, J. (2011). Relations between Executive Function and Academic Achievement from Ages 5 to 17 in a Large, Representative National Sample. Learning Individual Differences, 21(4), 327-336. doi:10.1016/j.lindif.2011.01.007

Bialystok, E. (1988). Levels of bilingualism and levels of linguistic awareness. Developmental Psychology, 24(4), 560-567. doi: 10.1037/0012-1649.24.4.560

Bryce, D., Whitebread, D. y Szücs, D. (2015). The relationships among executive functions, metacognitive skills and educational achievement in 5 and 7 year-old children. Metacognition and Learning, 10(2), 181-198. doi: 10.1007/s11409-014-9120-4

Casas, S. (2013). Relación entre las funciones ejecutivas y el rendimiento académico en la educación de adultos (tesis de maestría inédita). Universidad Internacional de La Rioja, La Rioja, España. Recuperado de https://reunir.unir.net/bitstream/handle/123456789/ 3008/CatalinaMaria_Martorell_Mir.pdf?sequence=1\&isAllowed=y

Casey, B. J., Jones, R. y Somerville, L. (2011). Braking and Accelerating of the Adolescent Brain. Journal of Research on Adolescence, 21(1), 21 - 33. doi: 10.1111/j.15327795.2010.00712.x 
Caso-Niebla, J. y Hernández-Guzmán, L. (2010). Modelo Explicativo del Bajo Rendimiento Escolar: Un Estudio con Adolescentes Mexicanos. Revista Iberoamericana de Evaluación Educativa, 3(2), 145-159. Recuperado de https://www.researchgate.net/publication/277271374_Modelo_Explicativo_del_Baj o_Rendimiento_Escolar_Un_Estudio_con_Adolescentes_Mexicanos

Flores, J.C., Ostrosky-Solís, F. y Lozano, A. (2008). Batería de Funciones Frontales y Ejecutivas: Presentación. Revista Neuropsicología, Neuropsiquiatría y $\begin{array}{lllll}\text { Neurociencias, } & 8(1), & \text { 141-158. } & \text { Recuperado de }\end{array}$ https://dialnet.unirioja.es/descarga/articulo/3987630.pdf

Flores-Lázaro, J., Castillo-Preciado, R. y Jiménez-Miramonte, N. (2014). Desarrollo de funciones ejecutivas, de la niñez a la juventud. Anales de Psicología, 30(2), 463-473. Recuperado de https://www.redalyc.org/pdf/167/16731188009.pdf

Goldberg, E. (1986). Varieties of perseveration: A comparison of two taxonomies. Journal of Clinical and Experimental Neuropsychology, 8(6), 710-726. doi: $10.1080 / 01688638608405191$

Kerlinger, F. N. y Lee, H. B. (2002). Investigación del comportamiento. Métodos de investigación en ciencias sociales. México: McGraw-Hill.

Kuhn, D. (2006) Do Cognitive Changes Accompany Developments in the Adolescent Brain? Perspectives on Psychological Science, 1(1), 59-67. doi: 10.1111/j.1745-6924.2006. t01-2-.x

Luria, A. (1977/2000). Las funciones corticales superiores en el hombre (3ra ed.). México: Fontamara.

Magnusson, D. (1998). The logic and implications of a person-oriented approach. En Cairns, R. B., Bergman, L. R. y Kagan, J. (Eds.), Methods and models for studying the individual (pp. 33-82). London, U.K.: Sage Publications.

Marino, D. y Julián, C. (2010). Actualización en tests neuropsicológicos de funciones ejecutivas. Revista Argentina de Ciencias del Comportamiento, 2(1), 34-45. Recuperado de https://www.redalyc.org/articulo.oa?id=333427068004 
Rosselli, M., Jurado, M. B. y Matute, E. (2008). Las funciones ejecutivas a través de la vida. Revista Neuropsicología, Neuropsiquiatría y Neurociencias, 8(1), 23-46. Recuperado de https://dialnet.unirioja.es/descarga/articulo/3987451.pdf

Sandson, J. y Albert, M. (1984). Varieties of perseveration. Neuropsycologia, 6, 715-732. doi: 10.1016/0028-3932(84)90098-8

Scott, W.A. y Wertheimer, M. (1981). Introducción a la investigación en psicología. México: El Manual Moderno.

Secretaría de Educación Pública [SEP] (2012). Reporte de la Encuesta Nacional de Deserción en la Educación Media Superior. México. SEP-COPEEMS. Recuperado de http://www.sems.gob.mx/work/models/sems/Resource/10787/1/images/Anexo 6Reporte_de_la_ENDEMS.pdf

Stelzer, F. y Cervigni, M. (2011). Desempeño académico y funciones ejecutivas en infancia y adolescencia. Una revisión de la literatura. Revista de Investigación en Educación, 9(1), 148-156. Recuperado de http://reined.webs.uvigo.es/index.php/reined/ $\underline{\text { article/view/107/97 }}$

Vergara, M. I. (2011). Funciones ejecutivas y desempeño académico en estudiantes de primer año de psicología de la corporación universitaria minuto de dios, en Bello Antioquia (tesis de maestría inédita). Universidad de San Buenaventura, Medellín, Colombia. Recuperado de https://s3.amazonaws.com/academia.edu.documents/ 38439002/Funciones_Ejecutivas_Estudiante_Vergara_2011.pdf?AWSAccessKeyId =AKIAIWOWYYGZ2Y53UL3A\&Expires=1547574033\&Signature $=$ MxnuIZzgH8 mpUR\%2BFi7\%2FomSc6i8Y\%3D\&response-content$\underline{\text { disposition=inline } \% 3 \mathrm{~B} \% 20 \text { filename\%3DFunciones_Ejecutivas_Estudiante_Vergara }}$ .pdf

Welsh, M., Peterson, E. y Jameson, M. (2017). History of Childhood Maltreatment and College Academic Outcomes: Indirect Effects of Hot Execution Function. Frontiers in Psychology, 8, 1-13. doi: 10.3389/fpsyg.2017.01091 